\title{
FIXED/FREE FINAL TIME SIR EPIDEMIC MODELS WITH MULTIPLE CONTROLS
}

\author{
Iacoviello, D. ${ }^{*} \&$ Liuzzi, G. ${ }^{* *}$ \\ * "Sapienza” University of Rome, Dept.of Computer and System Sciences “A. Ruberti”, \\ Via Ariosto 25, 00185 Rome, Italy \\ ** Consiglio Nazionale delle Ricerche, Istituto di Analisi dei Sistemi e Informatica “A. Ruberti”, \\ Viale Manzoni 30, 00185 Rome, Italy \\ E-Mail: iacoviel@dis.uniroma1.it; liuzzi@iasi.cnr.it
}

\begin{abstract}
In this paper the possible advantages in introducing multiple controls in the analysis of epidemic models are investigated. Usually, only the susceptible or infected people are controlled by vaccination or by quarantine and/or medicine treatment; in this paper multiple controls, both for the susceptible and infected are considered. The problem is studied both in the case of fixed and free final time. Numerical results are considered for simulated data showing the effects of multiple controls and the rule of each parameters of the model. Also a simulation on real data regarding the course of measles in Africa is presented.

(Received in September 2007, accepted in March 2008. This paper was with the authors 1 month for 1 revision.)
\end{abstract}

Key Words: Optimal Control, Epidemic Control, Nonlinear Programming, Direct

Transcription Methods

\section{INTRODUCTION}

The literature on epidemics is extremely vast. One of the motivations for the study of mathematical models for epidemics is related to the possibility of evaluation of different control strategies. The decision about the best vaccination campaign [1, 2], screening and educational campaigns [3], resource allocation [4,5] can be supported by suitable modelling and analysis of control strategies. A particularly encouraging approach uses the optimal control theory; interesting reviews can be found in [6]. Analytical results on the optimal control problems for epidemics are extensively described in [7]; in that paper models with control by vaccination, quarantine, screening or health campaigns are studied with a rather general choice for the interaction function. Also the presence of latency is considered, both in case of control by vaccination and in case of control by quarantine and screening. Usually, the control is assumed constrained between zero and a maximum value; it takes into account limitations from financial and technical point of view and, more in general, of resource. This latter problem of limited resource allocation has been studied in particular in [4, 5]; in [4] multiple population subgroups are considered with the aim of minimizing the number of new infections and maximizing the number of quality-adjusted life years gained. In [5] the problem of optimal allocation of a limited resource among multiple noninteracting populations is analysed; it is shown that the optimal resource allocation depends on many factors including the size of the population, the state of the epidemic in each population before resources are allocated, the length of the time horizon. Applications of optimal control theory to specific diseases can be found in [8-12]. In [8] the optimal control is applied to the chemotherapy of human immunodeficiency virus (HIV) dealing with the problem of deciding when and how treatment should be initiated, whereas in [9] the effects of a preventive vaccine in the transmission dynamics of HIV infection is studied. Different control strategies (isolation, quarantine, closing schools, tracing contacts of diagnosed cases) are considered to 
control emerging infectious diseases like SARS in [10]; the quarantine and tracing contacts of diagnosed cases appear to be successful in reducing transmission. Also in [11] strategies for the study and control of the HIV infection are considered whereas the optimal control theory is applied in the control program for tuberculosis in [12]. In [11, 12] two controls are assumed: in the first paper, one control boosts the immune system and the other delays the HIV progression; the interaction of HIV and T-cells in the immune systems is analysed and the optimal controls represent drug treatment strategies. In [12] the two control efforts considered represent case finding and case holding efforts; the optimal control is applied to a system consisting in six ordinary differential equations, modelling a two-strain tuberculosis model: the state variables represent the susceptibles, two different categories for the infected but not infectious, two different categories for the infectious and one category for the treated. The papers $[11,12]$ are the first attempts to concern with multiple controls applied to epidemic model, meaning the aim to control all the state variables. In most of the relevant literature the control involves just one class of people, usually the susceptible or the infected; in this case it is shown [7] that the optimum is given by a maximum effort control at the beginning of the epidemic. Another important aspect in the study of epidemic model is related to the rule of the final time of the control effort. Usually it is assumed fixed and, if sufficiently small, uniqueness results for the optimal bounded control are established [11]. In [3] the optimal control of an epidemic by means of educational campaigns is considered; the total time of the campaign is assumed budge limited. The end of the epidemic outbreak is defined as the first instant such that the number of infective becomes less than one; in this case it is not necessary to deal with an infinite time horizon control problem. In [11] this latter problem is considered; it is shown that the infinite time-horizon vaccination and quarantine problems possess unique optimal solution given by a maximal effort control on some initial interval. Epidemiological models introduce the following notations: $S$ stands for susceptible, that is the class of people who are not infected, $I$ denotes the infected, $R$ denotes the removed, that means dead or immune people. Some models introduce also the infected that are not yet infectious and are denoted by $E$. Therefore the most common models are denoted by SIR or SEIR notations and can vary each other for the functions describing the relation between one class of people and the others. When also the quarantined class Q is considered the SIQ or SIQR models are analysed, as in [13], in which periodic solutions by Hopf bifurcation are studied. In [14] another class is introduced, the class C of the "cross-immune individuals" and a SIRC model is considered and analysed with respect to influenza A.

In this paper the optimal control problem for SIR-epidemic model is considered; in particular control effort both for the susceptible and for the infected is assumed. First the final instant of the control effort is assumed constant and a comparison between the situations in which just one control is present is analysed. Moreover the multiple control effort is studied also in the case in which the final instant is assumed unknown and is a variable to be minimized. In Section 2 the general model with multiple control effort is presented; in Section 3 and 4 the analysis of the multiple controls with fixed final instant and free final time is considered respectively. Numerical results are shown in Section 5, and a case study regarding the course of measle in Africa is analysed.

\section{THE SIR MODEL AND MULTIPLE CONTROLS}

Let us denote by $x(t)$ the susceptible in a population, by $y(t)$ the infected and by $z(t)$ the removed. Following the most popular description of the epidemics [7], the dynamics of the epidemics, with interaction function $f$ between the susceptible and the infected, can be written as,

$$
\dot{x}(t)=-f(x, y) \quad(1), \quad \dot{y}(t)=f(x, \quad y)-\gamma y \quad(2), \quad \dot{z}(t)=\gamma y
$$


with initial known state condition for the susceptible and infected,

$$
x(0)=x_{0} \quad y(0)=y_{0}
$$

In this description the parameter $\gamma>0$ denotes the rate of removal of the infected.

The introduction of the vaccination implies the presence in (1) of an additive term representing the effort for vaccination thus obtaining,

$$
\dot{x}(t)=-f(x, y)-g(x) u_{1}
$$

The introduction of treatment or isolation for the infected may be described by introducing the term $-h(x, y) u_{2}$ in (2) so that it becomes,

$$
\dot{y}(t)=f(x, y)-\gamma y-h(x, y) u_{2}
$$

Different choice for the interaction function $f$ have been proposed in the relevant literature; in this paper the choice of [6] is adopted,

$$
f(x, y)=\beta x y /(x+y), \quad \beta>0
$$

The controls $u_{1}$ and $u_{2}$ represent two different treatment strategies; for example, if $g \equiv 1$, $u_{1}$ is the rate of vaccination; if $h(x, y)=a+y /(x+y+b), a, b \in R, u_{2}$ may represent the isolation effort or screening efficiency. The choices considered in this paper are the following, as suggested in $[1,6]$,

$$
g(x)=x \quad h(x, y)=y
$$

The two efforts are assumed to be limited both from below and above, namely, $0 \leq a_{1} \leq u_{1} \leq b_{1}, 0 \leq a_{2} \leq u_{2} \leq b_{2}$. Let us denote these box constraints by

$$
q(u(t))=\left(u_{1}(t)-b_{1} \quad a_{1}-u_{1}(t) \quad u_{2}(t)-b_{2} \quad a_{2}-u_{2}(t)\right)^{T} \leq 0
$$

As far as the cost functional is concerned two different choices tied to the final time $t_{f}$ are assumed. Namely, should the final time be fixed a priori, then the following cost function is considered

$$
J\left(u_{1}, u_{2}\right)=\int_{0}^{t_{f}}\left[y(t)+c_{1} u_{1}^{2}(t)+c_{2} u_{2}^{2}(t)\right] d t
$$

which, when minimized, amounts to decreasing the number of infected people at the minimum effort expressed in quadratic form. Section 3 is devoted to the analysis of the fixed final time setting.

On the other hand, should the final time be free, then the cost function

$$
J\left(t_{f}\right)=t_{f}
$$

is assumed, along with an addition final state condition such as

$$
y\left(t_{f}\right) \leq A
$$

The choice (11) is appropriate since in this case the aim is to determine controls $u_{1}, u_{2}$ such that the infected are reduced below a given threshold within the minimum time. The analysis of the free final time case is carried out in Section 4.

\section{ANALYSIS OF THE MULTIPLE OPTIMAL CONTROLS WITH}

\section{FIXED FINAL TIME}

In this section the following problem is considered.

Problem formulation (P1): Given the system:

$$
\dot{x}(t)=-\beta x y /(x+y)-x u_{1} \quad \dot{y}(t)=\beta x y /(x+y)-\gamma y-y u_{2}
$$

with initial condition (4) and fixed final time $t_{f}$ find the controls $u_{1}^{o}, u_{2}^{o}$ satisfying the 
constraints (9), minimizing the cost index (10). For the optimal control problem (P1) it is possible to state a result concerning the optimal controls $u_{1}^{o}(t)$ and $u_{2}^{o}(t)$.

Theorem 1: The above optimal control problem has the following solution:

$$
\begin{aligned}
& u_{1}^{o}(t)=\left\{\begin{array}{ccc}
a_{1} & \text { if } & \lambda_{1}(t) x^{o}(t) / 2 c_{1} \leq a_{1} \\
\lambda_{1}(t) x^{o}(t) / 2 c_{1} & \text { if } & a_{1} \leq \lambda_{1}(t) x^{o}(t) / 2 c_{1} \leq b_{1} \\
b_{1} & \text { if } & \lambda_{1}(t) x^{o}(t) / 2 c_{1} \geq b_{1}
\end{array}\right. \\
& u_{2}^{o}(t)=\left\{\begin{array}{ccc}
a_{2} & \text { if } & \lambda_{2}(t) y^{o}(t) / 2 c_{2} \leq a_{2} \\
\lambda_{2}(t) y^{o}(t) / 2 c_{2} & \text { if } & a_{2} \leq \lambda_{2}(t) y^{o}(t) / 2 c_{2} \leq b_{2} \\
b_{2} & \text { if } & \lambda_{2}(t) y^{o}(t) / 2 c_{2} \geq b_{2}
\end{array}\right.
\end{aligned}
$$

where $\lambda_{1}$ and $\lambda_{2}$ are the adjoint variables satisfying the equations:

$$
\begin{aligned}
& \dot{\lambda}_{1}(t)=\left(\lambda_{1}(t)-\lambda_{2}(t)\right) \beta y^{2}(t) /(x(t)+y(t))^{2}+\lambda_{1}(t) u_{1}(t) \\
& \dot{\lambda}_{2}(t)=-1+\left(\lambda_{1}(t)-\lambda_{2}(t)\right) \beta x^{2}(t) /(x(t)+y(t))^{2}+\lambda_{2}(t) u_{2}(t)+\lambda_{2}(t) \gamma
\end{aligned}
$$

with final conditions:

$$
\lambda_{1}\left(t_{f}\right)=0 \quad \lambda_{2}\left(t_{f}\right)=0
$$

Proof: The form of the optimal solution (14) and (15) and of the adjoint equations (16) and (17) comes from the Pontryagin's maximum principle [15]. Let us introduce the Hamiltonian function:

$$
\begin{aligned}
& H=\lambda_{0}\left(y+c_{1} u_{1}^{2}+c_{2} u_{2}^{2}\right)+\lambda_{1}\left(-\beta x y /(x+y)-x u_{1}\right)++\lambda_{2}\left(\beta x y /(x+y)-\gamma y-y u_{2}\right) \\
& +\omega_{11}\left(u_{1}-b_{1}\right)+\omega_{12}\left(a_{1}-u_{1}\right)+\omega_{21}\left(u_{2}-b_{2}\right)+\omega_{22}\left(a_{2}-u_{2}\right)
\end{aligned}
$$

where $\omega_{11}(t), \omega_{12}(t), \omega_{21}(t), \omega_{22}(t) \geq 0$ are penalty multipliers such that:

$$
\begin{array}{lll}
\omega_{11}\left(u_{1}-b_{1}\right)=0 & \omega_{12}\left(a_{1}-u_{1}\right)=0 & \text { at } u_{1}^{o} \\
\omega_{21}\left(u_{2}-b_{2}\right)=0 & \omega_{22}\left(a_{2}-u_{2}\right)=0 & \text { at } u_{2}^{o}
\end{array}
$$

The singular case $\lambda_{0}=0$ cannot be realized, otherwise also $\lambda_{1}(t)=\lambda_{2}(t)=0$, which is impossible. Let us assume $\lambda_{0}=1$. We differentiate the Hamiltonian (19) with respect to the states $(x, y)$ :

$$
\begin{aligned}
& \dot{\lambda}_{1}(t)=-\partial H / \partial x=\left(\lambda_{1}(t)-\lambda_{2}(t)\right) \beta y^{2}(t) /(x(t)+y(t))^{2}+\lambda_{1}(t) u_{1}(t) \\
& \dot{\lambda}_{2}(t)=-\partial H / \partial y=-1+\left(\lambda_{1}(t)-\lambda_{2}(t)\right) \beta x^{2}(t) /(x(t)+y(t))^{2}+\lambda_{2}(t) u_{2}(t)+\lambda_{2}(t) \gamma
\end{aligned}
$$

Final conditions for the adjoint variables are given by (18), see [15].

The optimality conditions of the optimal control problem yield:

$$
0=\partial H / \partial u_{1}=2 c_{1} u_{1}-\lambda_{1} x+\omega_{11}-\omega_{12} \quad 0=\partial H / \partial u_{2}=2 c_{2} u_{2}-\lambda_{2} y+\omega_{21}-\omega_{22}
$$

so that, taking into account the bound constraints for the controls and the non-negativeness of the penalty functions, expressions (14) and (15) for the control variables are found.

The instants $\overline{t_{1}}$ and $\bar{t}_{2}$ in which $\lambda_{1}\left(\bar{t}_{1}\right) x^{o}\left(\bar{t}_{1}\right) / 2 c_{1}=b_{1}, \lambda_{1}\left(\bar{t}_{2}\right) x^{o}\left(\bar{t}_{2}\right) / 2 c_{1}=a_{1}$ are respectively the instant in which the control $u_{1}$ changes from the maximum allowed value $b_{1}$ to the values assumed by the function $\lambda_{1}(t) x^{o}(t) / 2 c_{1}$ and the instant in which the control $u_{1}$ changes from $\lambda_{1}(t) x^{o}(t) / 2 c_{1}$ to the minimum allowed value $a_{1}$. Similarly there are two instants $\tilde{t}_{1}$ and $\tilde{t}_{2}$ in 
which the control $u_{2}$ changes from the maximum allowed value $b_{2}$ to the values assumed by the function $\lambda_{2}(t) y^{o}(t) / 2 c_{2}$ and then to the minimum allowed value $a_{2}$. As observed in [11] and [12], the boundedness of the state and the structure of the differential system imply uniqueness of the optimal control provided that the fixed final time $t_{f}$ is small.

Proposition: The quantity $\lambda_{1} x$ is monotonically decreasing, $\forall \beta>0$; if the parameter $\beta>0$ is sufficiently small also the quantity $\lambda_{2} y$ is monotonically decreasing.

Proof: Substituting the expressions (13) and (16)-(17) in the quantity $d\left(\lambda_{1} x+\lambda_{2} y\right) / d t$ it can be easily derived that $\lambda_{1} x+\lambda_{2} y$ is monotonically decreasing; moreover it is zero at the final time $t_{f}$, from (18). It follows that $\lambda_{1} x+\lambda_{2} y>0, \forall t<t_{f}$. Analogously, using this property, it may be easily deduced that also $\lambda_{1} X$ is monotonically decreasing. This implies that the commutation instants for the control $u_{1}$ are such that $\overline{t_{1}}<\overline{t_{2}}$.

As far as the control $u_{2}$ is concerned, it can be obtained that:

$$
d\left(\lambda_{2} y\right) / d t=-y+\beta x y\left(\lambda_{1} x+\lambda_{2} y\right) /(x+y)^{2}
$$

and, if the parameter $\beta$ is sufficiently small, $\lambda_{2} y$ is monotonically decreasing: this means that also the commutation instants for the control $u_{2}$ are such that $\tilde{t}_{1}<\tilde{t}_{2}$.

\section{ANALYSIS OF THE MULTIPLE OPTIMAL CONTROLS WITH}

\section{FREE FINAL TIME}

Let us now consider the case in which the final time of the campaign is itself a variable $t_{f}>0$ of the optimal control problem. This setting is of interest since it may correspond to a situation in which it would be necessary to estimate the minimum final time of the entire strategy of control, from vaccine to quarantine within which the infected drop below a given threshold $A$.

Problem formulation (P2): Given the system (13), with initial condition (4) find the controls $u_{1}^{o}, u_{2}^{o}$ satisfying the constraints (9), and the final time $t_{f}>0$ satisfying (12), minimizing the cost index (11).

Theorem 2: The above optimal control problem has the following solution:

$$
\begin{aligned}
& u_{1}^{o}(t)=\left\{\begin{array}{lll}
a_{1} & \text { if } & \lambda_{1}(t) x(t)<0 \\
b_{1} & \text { if } & \lambda_{1}(t) x(t)>0
\end{array}\right. \\
& u_{2}^{o}(t)=\left\{\begin{array}{lll}
a_{2} & \text { if } & \lambda_{2}(t) y(t)<0 \\
b_{2} & \text { if } & \lambda_{2}(t) y(t)>0
\end{array}\right.
\end{aligned}
$$

where $\lambda_{1}$ and $\lambda_{2}$ are adjoint variables satisfying the equation:

$$
\begin{aligned}
& \dot{\lambda}_{1}(t)=\left(\lambda_{1}(t)-\lambda_{2}(t)\right) \beta y^{2}(t) /(x(t)+y(t))^{2}+\lambda_{1}(t) u_{1}(t) \\
& \dot{\lambda}_{2}(t)=\left(\lambda_{1}(t)-\lambda_{2}(t)\right) \beta x^{2}(t) /(x(t)+y(t))^{2}+\lambda_{2}(t) u_{2}(t)+\lambda_{2}(t) \gamma
\end{aligned}
$$

with transversality conditions:

$$
\lambda_{1}\left(t_{f}^{o}\right)=0 \quad \lambda_{2}\left(t_{f}^{o}\right)=0 \quad H\left(t_{f}^{o}\right)=0
$$

Proof: In this case the Hamiltonian, in the significant situation of $\lambda_{0}=1$, is given by:

$$
\begin{aligned}
H\left(x, y, u_{1}, u_{2}\right)= & 1+\lambda_{1}\left(-\beta x y /(x+y)-x u_{1}\right)+\lambda_{2}\left(\beta x y /(x+y)-\gamma y-y u_{2}\right) \\
& +\omega_{11}\left(u_{1}-b_{1}\right)+\omega_{12}\left(a_{1}-u_{1}\right)+\omega_{21}\left(u_{2}-b_{2}\right)+\omega_{22}\left(a_{2}-u_{2}\right)
\end{aligned}
$$


where $\omega_{11}(t), \omega_{12}(t), \omega_{21}(t), \omega_{22}(t) \geq 0$ are penalty multipliers satisfying (20) and (21).

The necessary conditions are given by:

$$
\begin{aligned}
& \dot{\lambda}_{1}(t)=-\partial H / \partial x=\left(\lambda_{1}(t)-\lambda_{2}(t)\right) \beta y^{2}(t) /(x(t)+y(t))^{2}+\lambda_{1}(t) u_{1}(t) \\
& \dot{\lambda}_{2}(t)=-\partial H / \partial y=\left(\lambda_{1}(t)-\lambda_{2}(t)\right) \beta x^{2}(t) /(x(t)+y(t))^{2}+\lambda_{2}(t) u_{2}(t)+\lambda_{2}(t) \gamma
\end{aligned}
$$

The final conditions (29) descend from the maximum principle [15]. The optimality conditions yield:

$$
0=\partial H / \partial u_{1}=-\lambda_{1} x+\omega_{11}-\omega_{12} \quad 0=\partial H / \partial u_{2}=-\lambda_{2} y+\omega_{21}-\omega_{22}
$$

so that, taking into account the bound constraints for the controls and the non-negativeness of the penalty functions, expressions (25) and (26) for the control variables are found.

\section{NUMERICAL RESULTS}

Methods for solving optimal control problems can be classified as either direct methods or in direct methods $[16,17]$. What distinguishes the two approaches is the way they look for a solution of the optimal control problem. An indirect method attempts to solve the optimal control necessary conditions (22)-(24), for the fixed final time, or (31)-(33) for the free final time. So that, for instance in the case of fixed final time, it is necessary for an indirect method to explicitly derive the adjoint equations (22), (23), the control equations (24) and all of the transversality conditions (18). In contrast, a direct method does not require explicit derivation and construction of the necessary conditions. A direct method does not construct the adjoint equations, control equations and any of the transversality conditions. In the context of optimal control both the above approaches have been used (see, for example, $[12,16,18])$. The major disadvantage of using an indirect method is that, even if feasible state and control solutions are known a priori, there is no guarantee that the computed solution will improve on the known one. Moreover, an indirect method needs to guess values for the adjoint variables and to numerically solve the adjoint equations which can be very ill-conditioned in practice [19]. For this reason we employed a direct collocation method to numerically solve the optimal control problems of the preceding sections. Here we will consider some numerical experiments which are needed to analyse the effective usefulness of the double control over both the susceptible and the infected individuals. First we consider the case in which the final time $t_{f}$ is fixed and then the problem in which the final time $t_{f}$ is free, so that it is a variable of the problem. Both the resulting optimal control problems are infinite dimensional in that the control and state variables are functions of the time $t$. To overcome the difficulty related to the infinite-dimensionality, all the approaches to the solution of an optimal control problem are based on the idea of solving a finite dimensional approximation to it. To obtain such an approximation, we adopt a direct transcription method based on a trapezoidal rule which allows us to "discretize" the optimal control problem. The resulting problem is a finite dimensional constrained nonlinear programming problem (NLP). Every transcription method is based on the idea of subdividing the so-called phase duration interval $\left[0, t_{f}\right]$ into $n-1$

segments or subintervals of equal length $\Delta t$ by placing $t_{0}, t_{1}, \ldots t_{n-1}$ node, mesh or grid points such that $0=t_{0}<t_{1}<\ldots<t_{n-1}=t_{f}$. We consider the grid points to be equally distributed over the phase duration $\left[0, t_{f}\right]$. Thus, by posing $\Delta t=t_{f} /(n-1)$, the grid points can be expressed as $t_{k}=k \Delta t$ for $k=0, \ldots, n-1$. Note that, $t_{0}=0$ and $t_{n-1}=t_{f}$. Let us denote by $x_{k}=x\left(t_{k}\right), y_{k}=y\left(t_{k}\right)$, $u_{1, k}=u_{1}\left(t_{k}\right), u_{2, k}=u_{2}\left(t_{k}\right)$, for $k=0, \ldots, n-1$, the value of the state and control variables on the grid points. According to the trapezoidal rule, (13) can be approximated by:

$$
\xi\left(x_{k}, y_{k}, x_{k+1}, y_{k+1}, u_{1, k+1} ; \Delta t\right)=0 \quad \zeta\left(x_{k}, y_{k}, x_{k+1}, y_{k+1}, u_{2, k+1} ; \Delta t\right)=0
$$


$k=0, \ldots, n-2$, where $\xi\left(x_{k}, y_{k}, x_{k+1}, y_{k+1}, u_{1, k+1} ; \Delta t\right)$ and $\zeta\left(x_{k}, y_{k}, x_{k+1}, y_{k+1}, u_{2, k+1} ; \Delta t\right)$ are the defects of the approximation and are given by:

$$
\begin{aligned}
& \xi\left(x_{k}, y_{k}, x_{k+1}, y_{k+1}, u_{1, k+1} ; \Delta t\right)=x_{k+1}-x_{k}-\frac{\Delta t}{2}\left(-\beta \frac{x_{k} y_{k}}{x_{k}+y_{k}}-x_{k} u_{1, k}-\beta \frac{x_{k+1} y_{k+1}}{x_{k+1}+y_{k+1}}-x_{k+1} u_{1, k+1}\right) \\
& \zeta\left(x_{k}, y_{k}, x_{k+1}, y_{k+1}, u_{2, k+1} ; \Delta t\right)=y_{k+1}-y_{k}- \\
& \frac{\Delta t}{2}\left(\beta \frac{x_{k} y_{k}}{x_{k}+y_{k}}-\gamma y_{k}-y_{k} u_{2, k}+\beta \frac{x_{k+1} y_{k+1}}{x_{k+1}+y_{k+1}}-\gamma y_{k+1}-y_{k+1} u_{2, k+1}\right)
\end{aligned}
$$

$k=0, \ldots, n-2$.

Equations (34) impose that all the defects of the discrete approximation of (13) be zero. We placed $n=200$ grid points to carry out the above transcription and used the interior point nonlinear programming solver KNITRO v. 4.0 [20, 21] to compute a solution of the transcribed problem. This solver replaces the (NLP) by a series of barrier sub-problems controlled by a barrier parameter. The algorithm uses trust regions and a merit function to promote convergence to a solution of the problem. The algorithm performs one or more minimization steps on each barrier problem, then decreases the barrier parameter and repeats the process until the original problem has been solved to the desired accuracy.

\subsection{The fixed final time case}

In this case we consider optimal control problem (P1). The cost functional in (10) can be approximated by the following summation:

$$
\sum_{\mathrm{k}=0}^{n-1}\left[y_{k}+c_{1} u_{1, k}^{2}+c_{2} u_{2, k}^{2}\right] \Delta t
$$

and by using (34) we can thus consider the finite dimensional nonlinear constrained problem:

$$
\begin{array}{lll}
\min _{\substack{x_{k}, y_{k}, u_{1, k}, u_{2, k}, k=0, \ldots, n-1}} \sum_{k=0}^{n-1}\left[y_{k}+c_{1} u_{1, k}^{2}+c_{2} u_{2, k}^{2}\right] & \\
\text { s.t. } \quad \xi\left(x_{k}, y_{k}, x_{k+1}, y_{k+1}, u_{1, k+1} ; \Delta t\right)=0, & \forall k=0, \ldots, n-2 \\
\quad \zeta\left(x_{k}, y_{k}, x_{k+1}, y_{k+1}, u_{2, k+1} ; \Delta t\right)=0, & \forall k=0, \ldots, n-2 \\
a_{1} \leq u_{1, k} \leq b_{1}, \quad a_{2} \leq u_{2, k} \leq b_{2}, & \forall k=0, \ldots, n-1 \quad x_{0}=x(0), \quad y_{0}=y(0) .
\end{array}
$$

It is possible to show [18] that the necessary optimality condition for Problem (36) approximate in the limit, as $n \rightarrow \infty$, the necessary optimality condition for Problem (35) given by the Pontryagin's maximum principle [15]. The following choices for the parameters of the model are assumed: $\beta=0.044, \gamma=0.1, a_{1}=0, a_{2}=0, b_{1}=0.2, b_{2}=0.1, c_{1}=1, c_{2}=10$.

The initial conditions are $x(0)=200, y(0)=50$ and the final time $t_{f}$ is set equal to 80 . These choices are reasonable, for example, for the HIV immunology model [11] or for the Tuberculosis model [12].

Fig. 1 a) reports the behaviour of the optimal control $u_{1}^{O}$ along with the function $\lambda_{1}(t) x^{o}(t) / 2 c_{1}$ which shows that the computed solution satisfies relation (14). Analogously, Fig. 1 b) reports the behaviour of $u_{2}^{O}$ and $\lambda_{2}(t) y^{o}(t) / 2 c_{2}$. Again, the computed solution satisfies (15). It results that the controls $u_{1}^{O}$ and $u_{2}^{O}$ remain at their maximum value for a while, then, decrease monotonically to their minimum allowed value. 


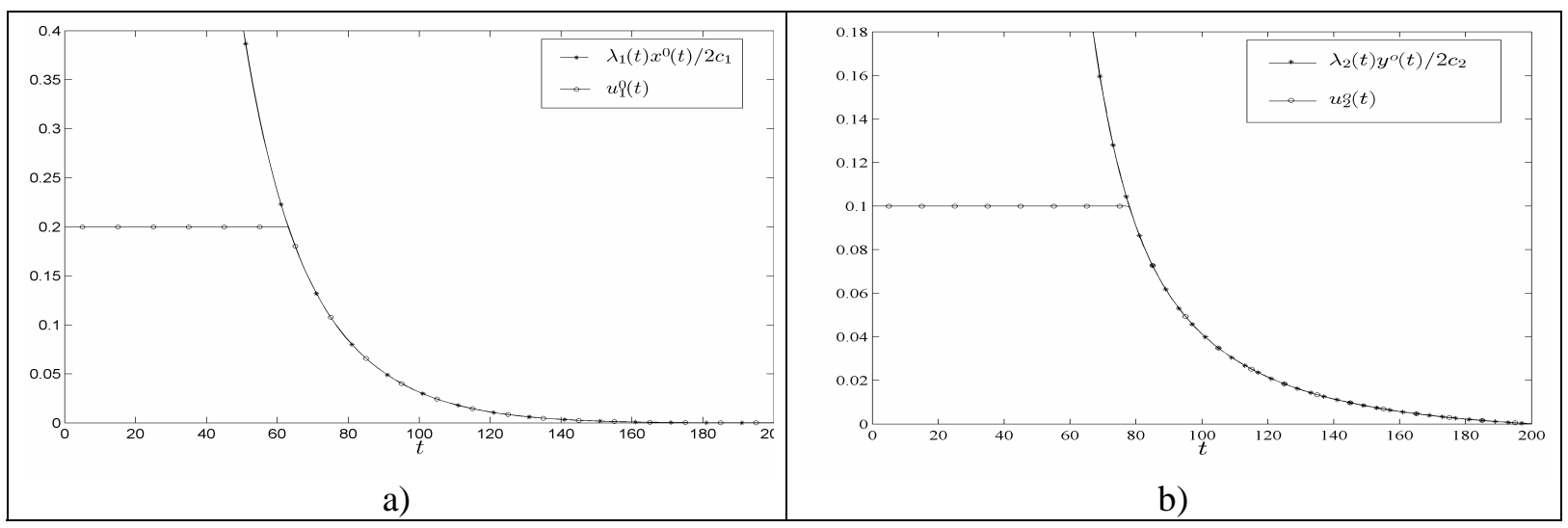

Figure 1: Behaviour of the optimal controls:

a) behaviour of the optimal control $u_{1}^{O}$ along with $\lambda_{1}(t) x^{o}(t) / 2 c_{1}$,

b) behaviour of the optimal control $u_{2}^{O}$ along with $\lambda_{2}(t) y^{o}(t) / 2 c_{2}$.

Fig. 2 reports the behaviour of the state variables $x^{0}(t)$ (Fig. 2 a) and $y^{0}(t)$ (Fig. 2 b) at the optimum. The best behaviour of the susceptibles and of the infected in case of multiple controls may be appreciated considering the situation in which just one control is assumed.

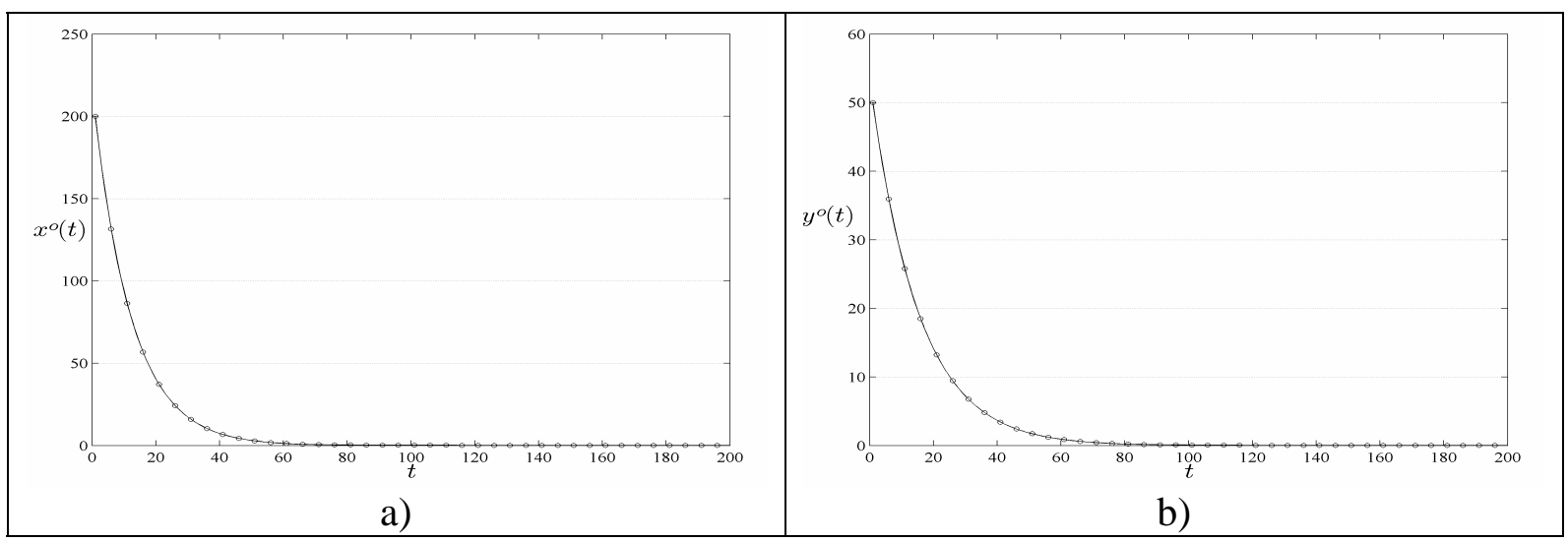

Figure 2: State variables at the optimum,

a) susceptibles $x^{0}(t)$ at the optimum,

b) infected $y^{0}(t)$ at the optimum.

In Fig. 3 a) the behaviour of the susceptible when only the susceptibles are controlled is plotted versus the behaviour of the susceptible obtained when only the infected are controlled. Analogously, Fig. 3 b) reports the behaviour of the infected when only the infected are controlled and when only the susceptibles are controlled. In order to show the influence that parameters $\beta$ and $\gamma$ have on the dynamic of susceptible and infected individuals respectively, optimal control problems for different values of these parameters are solved. In particular, Fig. 4 a) reports the dynamic of the susceptibles when parameter $\gamma=0.1$ and parameter $\beta=0.001,0.01,0.044$. Whereas, Fig. $4 \mathrm{~b}$ ) shows the behaviour of the infected when parameter $\beta=0.044$ and parameter $\gamma=0.1,0.05,0.01$. It is worth noting that, on the basis of numerical experiments, $y^{0}(t)$ is numerically insensitive to changes of the parameter $\beta$ just like $x^{0}(t)$ is not affected by changes of the parameter $\gamma$. 


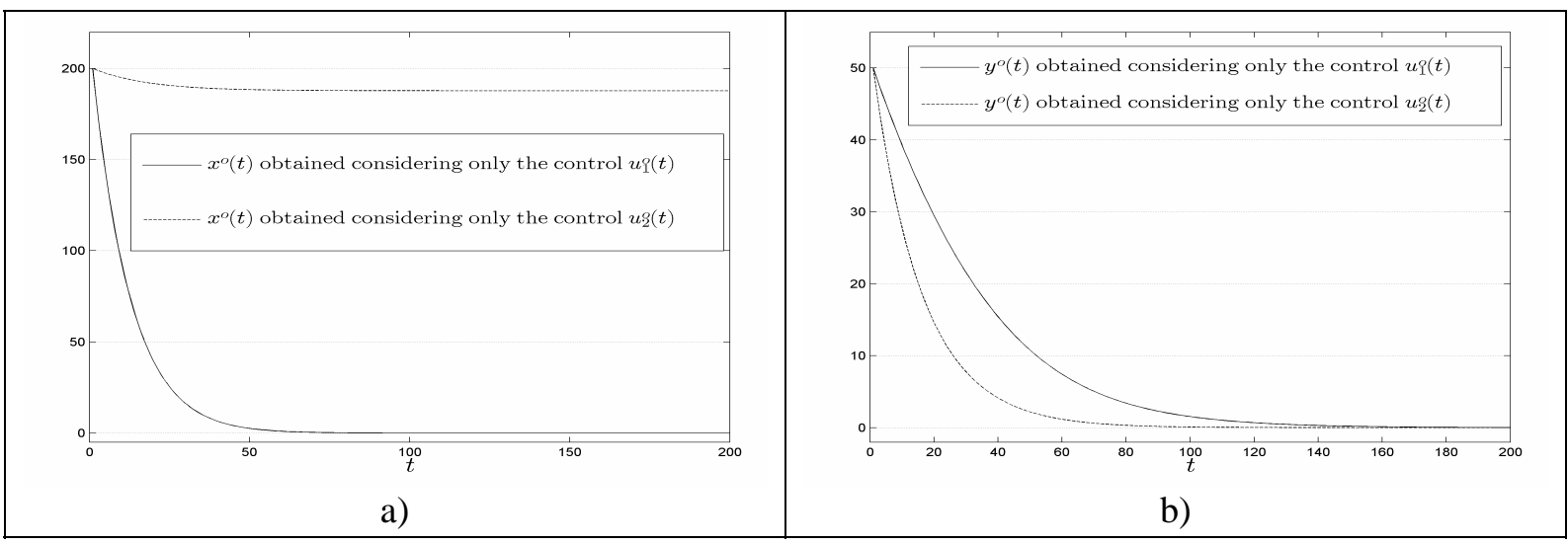

Figure 3: Behaviour of the susceptible and infected assuming just one control, a) behaviour of the susceptibles, b) behaviour of infected.

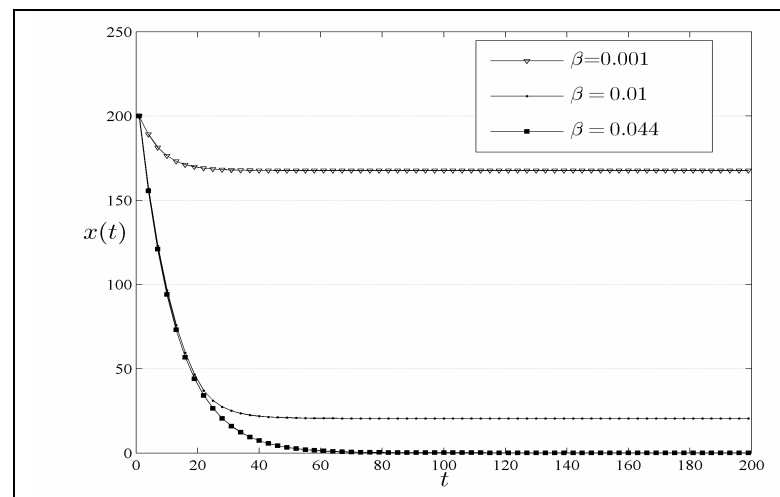

a)

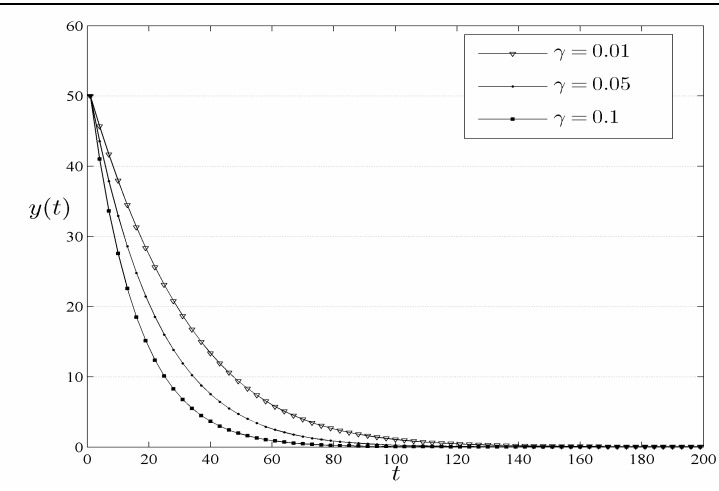

b)

Figure 4: Analysis of the influence of model parameters,

a) dynamic of the susceptibles when $\gamma=0.1$ with different values of $\beta$,

b) behaviour of the infected when $\beta=0.044$ with different values of $\gamma$.

\subsection{The free final time case}

Now we consider the case when the final time $t_{f}$ is free and is therefore itself a variable of the optimal control problem. In this particular case, given the definition of phase duration, we can write $t_{f}=(n-1) \Delta t$. Thus, the fact that $t_{f}$ is free implies that the phase duration $\Delta t$ is no more a fixed quantity but is itself a variable of the problem. In this case of free final time the optimal control problem (P2) is considered. This problem differs from the above problem (40) for the presence of $t_{f}$ in the cost functional and for the further requirement that the number of infected individuals at the final time be zero. The cost functional in Problem (P2) can be approximated by:

$$
\sum_{\mathrm{k}=0}^{n-1} \Delta t=n \Delta t
$$

and by using (34) the following finite dimensional nonlinear constrained problem can be considered:

$$
\min _{\substack{x_{k}, y_{k}, u_{1, k}, u_{2, k}, \Delta t \\ k=0, \ldots, n-1}} n \Delta t
$$

s.t. $\xi\left(x_{k}, y_{k}, x_{k+1}, y_{k+1}, u_{1, k+1}, \Delta t\right)=0$, 


$$
\begin{aligned}
& \zeta\left(x_{k}, y_{k}, x_{k+1}, y_{k+1}, u_{2, k+1}, \Delta t\right)=0, \quad \forall k=0, \ldots, n-2 \\
& a_{1} \leq u_{1, k} \leq b_{1}, \quad a_{2} \leq u_{2, k} \leq b_{2} . \quad \forall k=0, \ldots, n-1, x_{0}=x(0), \quad y_{0}=y(0), \quad y_{n-1} \leq A .
\end{aligned}
$$

where we set $A=0.01, a_{1}=a_{2}=0$ and $x(0)=200, y(0)=50$. In order to show the dependence of the optimal final time from the maximum allowed efforts, we solved problem (38) six times with increasing values for the upper bounds on the controls $b_{1}$ and $b_{2}$. In particular, for increasing values of $b_{1}$ and $b_{2}$ the following values of $t_{f}$ are obtained.

Table I: Values of final instants $t_{f}$ obtained solving problem (38) for different values of the upper bounds on the controls.

\begin{tabular}{|c|c|c|c|c|c|c|}
\hline$b_{1}, b_{2}$ & $0.2, \quad 0.1$ & $0.4,0.3$ & $0.6,0.5$ & $0.8,0.7$ & $1.0,0.9$ & $1.2,1.1$ \\
\hline$t_{f}$ & 48.70 & 23.07 & 15.01 & 11.11 & 8.81 & 7.30 \\
\hline
\end{tabular}

Accordingly, the experimentation pinpointed that the minimum final time decreases as the upper bounds increase. In Fig. 5, it can be noted that both the susceptibles and the infected decrease more quickly (smaller $t_{f}$ ) with greater upper bound for the controls; it means that with greater values for the upper bounds $b_{1}$ and $b_{2}$ the controls can assume a greater values.

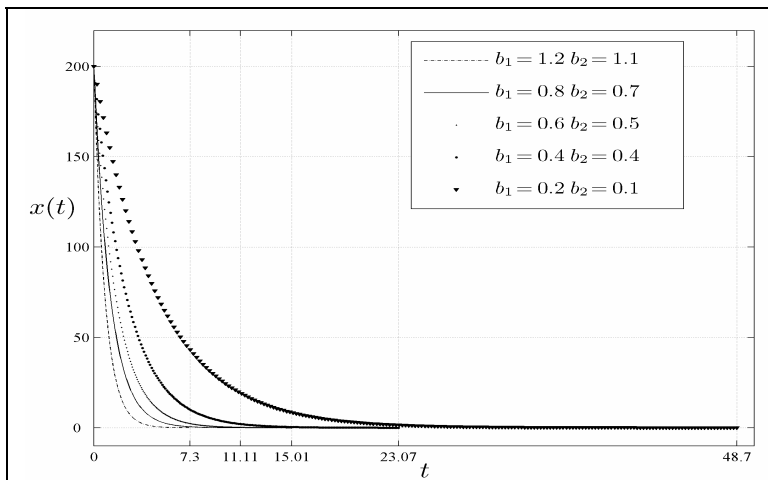

a)

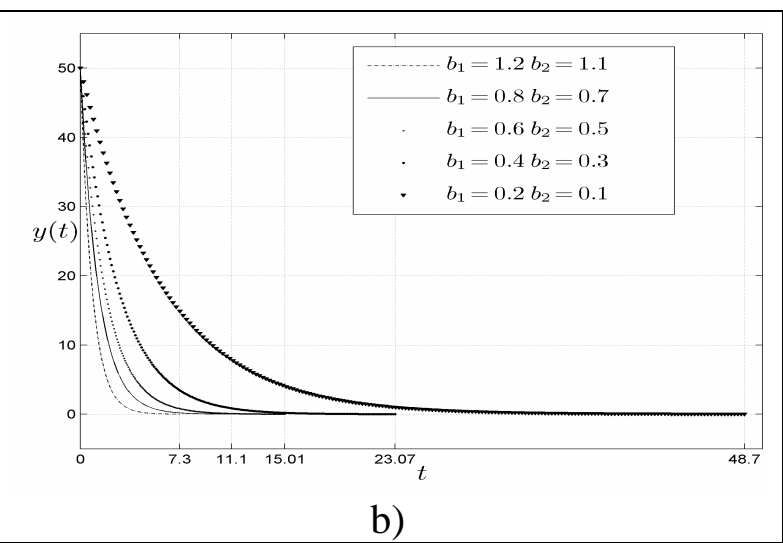

b)

Figure 5: Behaviour of the state variables when varying the upper bound of the controls, a) behaviour of the susceptibles, $\quad$ b) behaviour of the infected.

\section{CASE STUDY}

In order to test the effectiveness of the double control strategy, we have considered a real epidemic control problem. The data are taken from the World Health Organization website and concerns the course of measle in Africa, in the period between 1980 and 2005.

Table II: Number of people infected by measle in Africa, between 1980 and 2005.

\begin{tabular}{|c|c|c|c|c|c|c|c|}
\hline $\boldsymbol{t}_{\boldsymbol{i}}$ (year) & $\boldsymbol{y}_{\boldsymbol{i}}$ & $\boldsymbol{t}_{\boldsymbol{i}}$ (year) & $\boldsymbol{y}_{\boldsymbol{i}}$ & $\boldsymbol{t}_{\boldsymbol{i}}$ (year) & $\boldsymbol{y}_{\boldsymbol{i}}$ & $\boldsymbol{t}_{\boldsymbol{i}}$ (year) & $\boldsymbol{y}_{\boldsymbol{i}}$ \\
\hline 1980 & 1240993 & 1987 & 641057 & 1994 & 420193 & 2001 & 492116 \\
\hline 1981 & 1413184 & 1988 & 604244 & 1995 & 362925 & 2002 & 286380 \\
\hline 1982 & 1342685 & 1989 & 561896 & 1996 & 484914 & 2003 & 403572 \\
\hline 1983 & 1346883 & 1990 & 481204 & 1997 & 299623 & 2004 & 220732 \\
\hline 1984 & 1076106 & 1991 & 446517 & 1998 & 373149 & 2005 & 316224 \\
\hline 1985 & 1142002 & 1992 & 581125 & 1999 & 486660 & & \\
\hline 1986 & 676757 & 1993 & 395025 & 2000 & 520102 & & \\
\hline
\end{tabular}


During this period a great effort has been devoted to improve the number of vaccination so that, the data may be considered to refer to a model in which the major control was directed to the susceptibles that is to say $u_{2}=0$. Let us suppose that the vaccination campaign adopted in Africa in the considered period follows the optimal control obtained by solving problem P1 fixing $b_{2}=0$. When $b_{2}$ is fixed, Problem P1 depends on the parameters $c_{1}, c_{2}, a_{1}$, $a_{2}, x_{0}, y_{0}, \beta$ and $\gamma$. We set $c_{1}=1, c_{2}=10, b_{1}=0.2, a_{1}=a_{2}=0, x_{0}=14,000,000$ and $y_{0}=1,400,000$. Parameters $\beta$ and $\gamma$ are unknown and are determined in such a way that $y^{0}\left(t_{i}\right)$, $i=0, \ldots, 26$, approximates as well as possible $y_{i}$, for $i=0, \ldots, 26$. To this aim, we solve the least squares problem:

$$
\min \sum_{\mathrm{i}=1}^{26}\left(y^{0}\left(t_{i} ; \beta, \gamma\right)-y_{i}\right)^{2} \quad \text { s.t. } \quad 0.001 \leq \beta \leq 1 \quad 0.001 \leq \gamma \leq 0.7
$$

where $y^{0}\left(t_{i} ; \beta, \gamma\right), i=0, \ldots, 26$, are the sampled values at time instants $t_{i}$ of the infected state solution of Problem P1 for the given parameters $\beta$ and $\gamma$. Every objective function evaluation of Problem (39) requires the solution of the optimal control problem P1 which implies that the derivatives of the objective function are not available. For this reason, we solve Problem (39) by using the derivative-free minimization algorithm [22]. The optimal parameter values which allow for a better approximation of the real data in the minimum least squares sense are $\beta^{*}=0.0044$ and $\gamma^{*}=0.05$. Fig. 6 a) reports the real normalized data along with the results of the simulation showing an accordance between $y^{0}\left(t_{i} ; \beta^{*}, \gamma^{*}\right)$ and $y_{i}, i=0, \ldots, 26$. It is now possible to consider the complete model; that is the case when controls over both the susceptibles and infected are present, and solve the corresponding optimal control problem. In Fig. $6 \mathrm{~b}$ ) the real number of infected is plotted versus the number of infected that should have occurred if multiple controls were adopted. As it can be noted in Fig. 6 b), if in the years 1980-2005 a greater effort had been devoted both to vaccination and treatment/isolation, the infected should have decreased more quickly than in the real case.

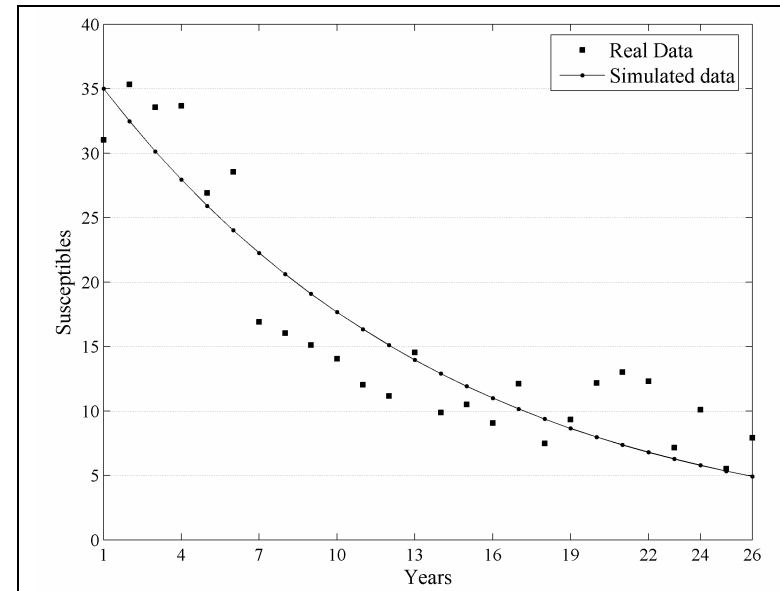

a)

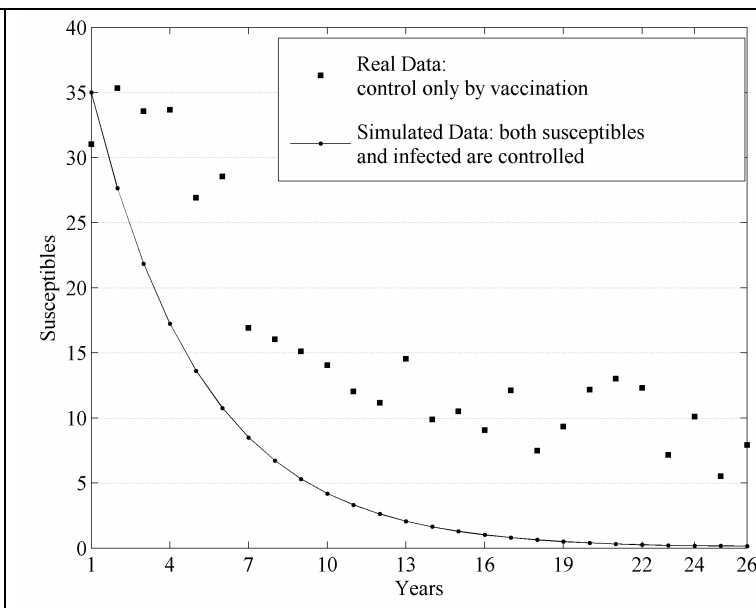

b)

Figure 6: Analysis of real data, a) real normalized data and result of simulation,

b) real data, in which the population is controlled only by vaccination, versus the simulation result in which both the susceptibles and the infected are controlled.

\section{CONCLUSIONS}

The analysis of SIR epidemic model in the presence of multiple controls is studied. In usual models just the susceptible or the infected are controlled by either vaccination or quarantine or medicine. The presence of the multiple controls was studied both in case of fixed final time 
and in the case in which the final time is a variable to be determined. Numerical results are presented showing that the presence of the double controls will diminish the number of susceptible and infected in a minor time than in case of a single control.

\section{REFERENCES}

[1] Verriest, E.; Delmotte, F.; Egerstedt M. (2005). Control of epidemics by vaccination, Proceedings of the American Control Conference

[2] Becker, N. G.; Starczak, D. N. (1998). Optimal vaccination strategies for a community of household, Mathematical Biosciences, Vol. 139, 117-132

[3] Castilho, C. (2006). Optimal control of an epidemic trough educational campaign, Electronic Journal of differential equations, Vol. 125, 1-11

[4] Zaric, G. S.; Brandeau, M. (2002). Dynamic resource allocation for epidemic control in multiple populations, Journal of Mathematics Applied in Medicine and Biology, Vol. 19, 235-255

[5] Brandeau, M.; Zaric, G. S.; Richter, A. (2003). Resource allocation for control of infectious diseases in multiple independent populations: beyond cost-effectiveness analysis, Journal of health economics, Vol. 22, 575-598

[6] Clancy, D.; Piunovskiy, A. B. (2005). An explicit optimal isolation policy for a deterministic epidemic model, Applied mathematics and computation, Vol. 163, 1109-1121

[7] Behncke, H. (2000). Optimal control of deterministic epidemics, Optimal control applications and methods, Vol. 21, 269-285

[8] Kirschner, D.; Lenhart, S.; Serbin, S. (1997). Optimal control of the chemotherapy of HIV, Journal of Mathematical Biology, Vol. 35, 775-792

[9] Gumel, A. B.; Moghadas, S. M.; Mickens, R. E. (2004). Effect of a preventive vaccine on the dynamics of HIV transmission, Communications in Nonlinear science and numerical simulation, Vol. 9, 649-659

[10] Becker, N. G.; Glass, K.; Li, Z.; Aldis, G. K. (2005). Controlling emerging infectious diseases like SARS, Mathematical Biosciences, Vol. 193, 205-221

[11] Joshi, H. R. (2002). Optimal control of an HIV immunology model, Optimal control applications and methods, Vol. 23, 199-213

[12] Jung, E.; Lenhart, S.; Feng, Z. (2002). Optimal control of treatment in a two strain tubercolosis model, Discrete and continuous dynamical systems-SERIES B, Vol. 2, 473-482

[13] Hethcote, H.; Zhien, M.; Shengbing, L. (2002). Effects of quarantine in six endemic models for infectious diseases. Mathematical Biosciences, Vol. 180, 141-160

[14] Casagrandi, R., Bolzoni, L. Levin, S. A., Andreasen, V. (2006). The SIRC model and influenza A, Mathematical Biosciences, Vol. 200, 152-169

[15] Hartl, R. F.; Sethi, S. P.; Vickson, R. G. (1995). A survey f the maximum principles for optimal control problems with state constraints, SIAM, Vol. 37, 181-218

[16] Betts, J. T. (2001). Practical Methods for Optimal Control Using Nonlinear Programming, SIAM Society for Applied and Industrial Mathematics, Philadelphia

[17] Von Stryk, O.; Bulirsch, R. (1992). Direct and Indirect methods for trajectory optimization, Annals of Operations Research, Vol. 37, 357-373

[18] Von Stryk, O. (1993). Numerical Solution of Optimal Control Problems by Direct Collocation, in Optimal Control-Calculus of Variations, Optimal Control Theory and Numerical Methods, Bulirsch, R.; Miele, A.; Stoer, J.; Vell, K.-H. (eds.), International Series of Numerical Mathematics, Vol. 111, 129-143

[19] Bryson Jr, A. E.; Ho, Y.-C. (1975). Applied Optimal Control, John Wiley \& Sons, New York

[20] Byrd, R. H.; Hribar, M. E.; Nocedal, J. (1999). An interior point algorithm for large scale nonlinear programming, SIAM Journal on Optimization, Vol. 9, 877-900

[21] Byrd, R. H.; Nocedal, J.; Waltz, R. A. (2006). An Integrated Package for Nonlinear Optimization, in Large-Scale Nonlinear Optimization, di Pillo, G.; Roma, M. (eds.), SpringerVerlag, 35-59

[22] Lucidi, S.; Sciandrone, M. (2002). A Derivative-free Algorithm for Bound Constrained Optimization, Computational Optim. And Appl., Vol. 21, 119-142 